\title{
Corela
}

Cognition, représentation, langage

HS-12 | 2012

Langue, espace, cognition

\section{En passant par : une expression en voie de grammaticalisation?}

\section{Dejan Stosic}

\section{(2) OpenEdition}

\section{Journals}

Édition électronique

URL : http://journals.openedition.org/corela/2844

DOI : $10.4000 /$ corela.2844

ISSN : $1638-573 \mathrm{X}$

\section{Éditeur}

Cercle linguistique du Centre et de I'Ouest - CerLICO

\section{Référence électronique}

Dejan Stosic, «En passant par : une expression en voie de grammaticalisation ? », Corela [En ligne] HS-12 | 2012, mis en ligne le 19 décembre 2012, consulté le 30 avril 2019. URL : http:// journals.openedition.org/corela/2844 ; DOI : 10.4000/corela.2844

Ce document a été généré automatiquement le 30 avril 2019

\section{(c) (i) (2)(2)}

Corela - cognition, représentation, langage est mis à disposition selon les termes de la licence Creative Commons Attribution - Pas d'Utilisation Commerciale - Partage dans les Mêmes Conditions 4.0 International. 


\title{
En passant par : une expression en voie de grammaticalisation?
}

\author{
Dejan Stosic
}

1 L'objectif de cet article ${ }^{1}$ est d'étudier la structure en passant par à la fois en synchronie du français moderne et en diachronie ( $\mathrm{du} 16^{\mathrm{e}}$ au $21^{\mathrm{e}}$ siècle). Correspondant formellement au gérondif du verbe passer suivi de la préposition par, la séquence en passant par a connu une diversification des contextes d'emplois telle que son fonctionnement n'est plus exclusivement celui d'une forme gérondive décrivant le déplacement dans l'espace. Le contraste entre les exemples (1) et (2) le montre bien:

(1) Comme il se trouvait dans un quartier où il n'y avait que du menu peuple, en passant par une rue, il entendit qu'on parlait assez haut: il s'approcha de la maison d'où venait le bruit. (A. Galland, Les Mille et une Nuits, 1715) ${ }^{2}$

(2) Nous étions élevés dans le culte des hommes du "Grand Métier », ces pêcheurs d'Islande célébrés par une pléiade d'écrivains de Victor Hugo à Roger Vercel en passant par Pierre Loti et Joseph Conrad. (M. Tournier, Le Médianoche amoureux, 1989)

2 Parallèlement à cette extension d'emploi, on note une forte récurrence de en passant par en français contemporain, notamment dans le discours journalistique. La jonction de ces deux tendances laisse penser que cette structure est en train de subir un processus de figement qui, s'il se poursuit, aboutira à la formation d'une nouvelle unité lexicale dans la langue, le lexique étant pris ici au sens large du terme. Notre propos consistera à montrer que ce processus est déjà bien amorcé depuis plusieurs décennies et qu'il s'agit d'un cas de grammaticalisation, puisqu'un élément lexical, en l'occurrence l'une des formes du verbe passer accompagnée de par, est en voie de devenir un élément grammatical, à savoir une préposition complexe. Nous partirons ici de la définition originelle de la grammaticalisation, proposée par A. Meillet ([1912] 1948: 131) : «le passage d'un mot autonome au rôle d'élément grammatical ", mais nous serons amenés à en adopter une définition plus large en prenant en compte des études plus récentes sur ce phénomène (voir § 2.2.).

3 L'article se subdivise en deux parties. Dans la première section, nous avancerons deux hypothèses, la première posant la nécessité de distinguer les emplois «libres » et les 
emplois «figés » de en passant par, la deuxième postulant que cette séquence est en voie de grammaticalisation et que le résultat de cette évolution est l'apparition en français contemporain d'une nouvelle préposition. Les deux hypothèses seront discutées et justifiées dans la deuxième section, où nous les mettrons à l'épreuve des données. L'étude sur corpus fera ressortir des différences de fonctionnement entre les emplois du début de la période prise en compte et ceux du français moderne. Ces différences suggèrent une évolution assez nette qui aboutit à la coexistence, en français contemporain, du gérondif du verbe passer suivi d'un syntagme prépositionnel en par et de la forme figée en passant par, proche par son comportement des prépositions. De manière plus générale, cette analyse mettra au jour les conséquences sémantiques et fonctionnelles du changement catégoriel que la forme gérondive du verbe de déplacement passer est en train de subir.

\section{Hypothèses}

4 La première hypothèse que nous ferons ici est que, pour rendre compte de l'ensemble des emplois de la séquence en passant par en français moderne, il est indispensable d'en distinguer deux types: des emplois que l'on peut qualifier de «libres» et des emplois « figés ».

5 Concernant tout d'abord les emplois libres, illustrés en (3-5), ils peuvent être concrets (3) ou figurés (4-5). En (3), le verbe passer s'applique à son domaine primitif: il y exprime le déplacement d'un humain dans l'espace, alors qu'en 4 et 5, tout en gardant ses propriétés sémantiques, passer s'applique à deux autres domaines sémantiques :

(3) En passant par la ville, il était venu, suivant son habitude, embrasser sa sœur et son neveu (R. Rolland, Jean-Christophe : L'Adolescent, 1905)

(4) L'art décoratif s'insinue dans les demeures particulières en passant par les installations publiques. (P. Abraham (dir.), Arts et littérature dans la société contemporaine, 1935)

(5) Acer acquiert Packard Bell en passant par Gateway. (Internet)

6 Il est évident que les emplois libres de la séquence en passant par (3-5), qu'ils soient concrets ou figurés, correspondent au gérondif du verbe passer suivi d'un SPrép introduit par par, ce que l'on peut représenter comme suit :

(6) en passant par la ville $=$ en passant $[$ par la ville $]=\mathrm{V}$ [Prép $+\mathrm{SN}]$.

7 Même s'il y a une cohésion sémantique très forte entre passer et par, il n'y a pas de figement proprement dit. Certes, la préposition par fonctionne ici comme une sorte de " satellite du verbe » à cause du rapport privilégié que les deux éléments entretiennent du fait de leur compatibilité sémantique (cf. Aurnague 2000), mais le sens du verbe et de la préposition reste transparent, on observe la coréférence du sujet à chaque fois, etc. Dans ce type d'emploi, d'un point de vue strictement syntaxique, rien ne distingue les exemples (3-5) des exemples qui suivent, où le gérondif du verbe passer, au sens premier (7-10) ou figuré (11-12), est suivi d'une autre préposition :

(7) Au désespoir d'avoir manqué son coup, il enfila la porte du jardin d'Ali Baba, qui donnait dans la cour, et, de jardin en jardin, en passant par-dessus les murs, il se sauva. (A. Galland, Les Mille et une Nuits, 1715)

(8) Son blouson sur l'épaule, le bras nu dépassant de la chemise retroussée, je le vis arracher son pansement en passant devant une poubelle. (H. Guibert, À l'ami qui ne m'a pas sauvé la vie, 1990)

(9) C'est un peu avant Rioja que l'on prend à droite, en passant sur un pont. (A. T'Serstevens, L'Itinéraire espagnol, 1963) 
(10) Un peu plus tard, ayant lu la lettre de Paulhan (que nous avons trouvée à six heures, en passant à l'hôtel) il m'a dit: "Il va vous emmerder, ce type ». (J-P. Sartre, Lettres au castor et à quelques autres, 1983)

(11) Les pensées de Dieu perdent quelque chose de leur qualité divine en passant dans l'esprit des hommes. (A. Billy, Introibo, 1939)

(12) Il conta, en passant par-dessus les détails, qu'arrivé en Suisse, il s'était mis en rapport avec M. Karl. (J. Romains, La Douceur de la vie, 1939)

Ce type de variation est exclu dans les emplois qualifiés de "figés », qui se caractérisent par une très forte cohésion syntaxique et sémantique entre le gérondif du verbe passer et par. La séquence en passant par semble présenter un certain degré de figement ${ }^{3}$ et apparaît dans deux structures syntaxiques particulières, illustrées respectivement en (13) et (14) :

(13) D'Alexandre à Voltaire, d'Aristote à Marivaux en passant par Beccaria, Durkheim et Condorcet, de «Colonies " à "Vertu », d' " Athéisme » à "Sérail » en passant par «Despotisme», "Gallicanisme» et "Science», les notions fondamentales traitées par Montesquieu sont passées en revue. (Dictionnaire électronique Montesquieu)

(14) Les premières étapes ont été l'assurance, puis l'assistance. Les clients sont prêts maintenant à « l'assumance " c'est-à-dire qu'on les aide à assumer leurs problèmes de toute nature: des examens médicaux, en passant par les démarches administratives, le coaching ou même le relooking. La difficulté est que certains de ces services ne sont pas rentables pour le moment. (La Tribune, octobre 2010)

$9 \mathrm{Au}$ vu de ces exemples, et sans nous attarder pour l'instant sur cette première hypothèse qui sera justifiée dans la section qui suit, nous émettrons notre deuxième hypothèse : en emploi «figé » (13-14), en passant par ne fonctionne plus comme un verbe suivi d'une préposition, mais comme une locution prépositionnelle. La possibilité de remplacer, dans de nombreux cas, en passant par figé par via étaie cette hypothèse. Le parallélisme entre (13) et (15) est très net :

(15) Multiples pourraient être ses relations intertextuelles avec d'autres poissons rouges littéraires, de Gautier à Eluard via Balzac et Flaubert, mais c'est un extrait du finale de Bel Ami publié plus de dix ans après le roman de Zola (1885) qui retiendra notre attention. (Internet)

10 Tout semble indiquer, en effet, que la structure en passant par dans ce type d'emploi, par son comportement syntaxique et sémantique, est devenue une préposition, statut qui ne lui est reconnu ni par les lexicographes ni par les linguistes. En reprenant la représentation en (6), on obtient :

(16) en passant par Condorcet $=$ [en passant par $]$ Condorcet $=$ Prép $+\mathrm{N}$.

11 L'idée qui est sous-jacente à cette hypothèse est que l'expression en passant par est le résultat d'un processus de grammaticalisation assez avancé, c'est-à-dire qu'une forme verbale, en l'occurrence le gérondif du verbe passer, et son satellite par sont devenus un seul élément grammatical - une préposition. Nous validerons cette dernière hypothèse en deux étapes :

- en montrant que dans un état de langue antérieur il n'y avait que la structure gérondive et que les emplois de type (13) et (14) n'existaient pas.

- en justifiant l'analyse de en passant par comme une préposition en français moderne.

12 Avant de vérifier les deux hypothèses, précisons que, dans les dictionnaires et dans les travaux sur les prépositions, en passant par n'est pratiquement jamais répertorié comme locution, prépositionnelle ou autre. Par exemple, Le Petit Robert et le Lexis n'en font aucune mention. Le TLFi et Le Robert électronique, pour leur part, mentionnent passer par dans des emplois concrets et figurés qu'ils illustrent par des exemples comportant 
l'expression en passant par mais n'identifient pas celle-ci en tant que telle. On ne la trouve pas non plus dans Le dictionnaire des expressions et locutions de Rey \& Chantreau. De même, le $D H L F$ ne répertorie pas cette expression dans l'article consacré au verbe passer. C'est en fait essentiellement dans les dictionnaires bilingues (voir, entre autres, Sylang, Dictionnaire Larousse français-anglais) que en passant par figure comme expression, et plus précisément comme locution prépositionnelle ou préposition ${ }^{4}$. Dans les travaux sur les prépositions en français, ni Laur (1991), ni Borillo (1997, 1998), ni Melis (2003) ne répertorient en passant par parmi les prépositions ou locutions prépositionnelles. A notre connaissance, seule Halmøy dans son ouvrage sur le gérondif fait apparaître en passant par comme « figement prépositionnel ", à côté de en allant (à, sur, vers) et en partant (de) (cf. Halmøy $2003: 78$ ).

\section{Justification des hypothèses}

\subsection{De la nécessité de distinguer des emplois libres et figés de en passant par}

13 Selon notre première hypothèse, il y aurait lieu de distinguer en français contemporain les emplois libres et les emplois figés de la séquence en passant par. Plusieurs traits de son fonctionnement syntaxique et sémantique permettent d'opposer les deux types d'usages.

14 1) Tout d'abord, du point de vue lexico-sémantique, en emploi libre, on observe des contraintes de sélection très strictes sur le régime de la préposition. En effet, les contraintes qui pèsent sur le choix du complément de en passant par ne se distinguent en rien de celles qui sont imposées par la préposition par lorsqu'elle précédé du verbe passer : en pareille situation, seuls les SN susceptibles de compléter passer par peuvent compléter en passant par:

(17) a. Nous sommes passés par Paris.

b. Ils ont perdu beaucoup de temps en passant par Paris

(18) a. Notre société passe par une crise très grave.

b. Oui, notre société, notamment occidentale, est à repenser et reconstruire et cela ne pourra se faire qu'en passant par une crise majeure. (Internet, 2008)

(19) a. Pour accomplir sa mission, Jean-Pierre a dû passer par le responsable des relations internationales.

b. Jean-Pierre a accompli sa mission en passant par le responsable des relations internationales.

Voici quelques exemples de noms susceptibles d'apparaître dans le SN régime de par accompagnant le gérondif de passer :

- noms concrets : porte, avenue, ville, rue, foire, route, fenêtre, place, hôtel, ainsi que la grande majorité des toponymes.

- noms abstraits : néant, dépression, canal médiatique, phase(s), étape, crise, etc.

Les contraintes lexicales qui pèsent sur les emplois figés de en passant par sont, en revanche, beaucoup moins fortes, d'où la possibilité d'avoir comme complément un éventail beaucoup plus large de SN. C'est ce qui permet d'expliquer à la fois l'acceptabilité de (20a) et (21) et l'inacceptabilité de (20b), où le maintien des contraintes lexicales propres à passer par empêche le $\mathrm{SN}$ Le père Goriot d'apparaitre comme complément de la préposition :

(20) a. De L'étranger à La Peste de Camus en passant par Le père Goriot ou encore Germinal, des analyses détaillées des œuvres des plus grands auteurs de la langue 
française sont disponibles sur Fichesdelecture.com. (Internet)

b. *Nous avons étudié le roman du XIXe siècle en passant par Le père Goriot.

(21) De l'arabe au vietnamien, en passant par l'hébreu et le japonais, ce sont plus de 300 polices gratuites à télécharger directement à partir de notre serveur. (Internet)

L'étude détaillée des exemples rassemblés dans notre corpus suggère que la liste des noms susceptibles d'accompagner en passant par en emploi figé est quasiment infinie :

Aristote, Lamartine, Germinal, travailleurs, émeraude, noces, roi, fusion, sacrifice, états d'âme, néo-conservateurs, folie, amitiés, curiosité, carabine, nom, chef de poste, manipulation, ambulance, camion, bleu de Prusse, variété(s), République consulaire, nuque, dépression, etc.

2) Cette plus grande diversité lexicale des $\mathrm{SN}$ compléments est à mettre en rapport avec le statut syntaxique particulier de l'expression en passant par en emploi figé. En effet, en tant que séquence figée, en passant par apparaît en règle générale au sein d'une structure tripartite constituée d'un SPrép de polarité initiale introduit par (allant, partant) de ou depuis, d'un SPrép de polarité finale introduit par à ou jusqu'à et d'un troisième élément introduit par en passant par qui fixe un ou plusieurs jalons du parcours réel ou fictif délimité par les deux points extrêmes. Les exemples ci-dessous illustrent cette structure :

(22) La tradition substantialiste, d'Aristote aux modernes en passant par les nominalistes, privilégiait les noms et les adjectifs, lesquels expriment respectivement la substance et les attributs. (C. Hagège, L'Homme de paroles, 1985)

(23) Du débat sur les OGM à celui sur la loi HADOPI en passant par les discussions budgétaires, les épisodes agités n'ont pas manqué au Palais Bourbon. (La voix du nord, septembre 2010)

(24) Moderne, l'aéroport peut accueillir des appareils de tous types, allant des avions de ligne aux jets privés en passant par des cargos géants et l'Airbus A380, selon la même source. (Internet)

Dans ce type de contexte, en passant par présente les propriétés d'un élément fonctionnel, dont le rôle est d'incorporer un SN dans l'ensemble de la structure de la phrase, d'où la disparition des contraintes sémantiques propres à passer et à par. On peut se demander si l'évolution de la séquence en passant par en un élément fonctionnel est due complètement au hasard. Nous estimons que le passage du lexical au fonctionnel que connaît cet élément peut s'expliquer en bonne partie à partir du sens même de la structure combinant le verbe passer et la préposition par. Dans l'expression de l'espace, par est utilisé pour localiser une entité mobile pendant la phase médiane d'un déplacement impliquant, explicitement ou implicitement, deux ou plusieurs autres entités. D'après Aurnague \& Stosic (2002) et Stosic (2002), le sens spatial de la préposition par peut être défini à l'aide du concept de " trajet ». En effet, l'étude de la nature des prédicats verbaux et des noms susceptibles de se combiner avec par spatial nous a permis de montrer que cette préposition participe essentiellement à l'expression de procès téliques et que les entités localisatrices dénotées par le $\mathrm{SN}$ régime de la préposition se caractérisent par leur capacité à faciliter le passage et à mettre en relation, autant au niveau référentiel qu'au niveau pragmatique, les lieux initial et final du déplacement. Le verbe passer ne fait qu'accentuer le caractère transitoire de la relation établie entre l'entité localisée et l'entité localisatrice.

Nous estimons donc que le sémantisme même de cette structure ouvre des possibilités de figement. Plus précisément, c'est la fonction de « connexion » intrinsèque au sémantisme de la préposition par, étayée par celui du verbe passer, qui pourrait expliquer en bonne partie comment ${ }^{5}$ la séquence en passant par a évolué des emplois libres/lexicaux vers les 
emplois figés/fonctionnels. Il s'agirait, selon nous, d'un mouvement allant de l'expression de la mise en relation (sémantique) des points extrêmes d'un trajet à la prise en charge d'une mise en relation des points extrêmes d'un tout ordonné à un niveau plus abstrait, y compris syntaxique. La structure tripartite serait dans ce cas la transposition en syntaxe de la structure conceptuelle sous-jacente à la notion de «trajet ». Dès lors, sans exprimer un trajet quelconque, en passant par permet d'insérer dans la phrase l'un des éléments d'un ensemble relativement ordonné. Cet élément se situe nécessairement à l'intérieur d'un tout a priori linéaire dont les limites sont fixées par des SPrép initial et final. La nécessaire inscription de l'élément introduit par en passant par dans un tout est souvent soulignée par la présence, dans le contexte gauche ou droit, d'unités comme: tout (déterminant ou pronom), partout, (vaste) gamme/éventail/série, etc. :

(25) Quant aux assemblages eux-mêmes, toute une gamme en est offerte, depuis les assemblages bois sur bois jusqu'aux assemblages à clous ou à aiguilles en passant par les différents assemblages par boulons. (Internet)

(26) Ce qui rend Leuwarden agréable et toute curieuse, c'est qu'elle a conservé la bonne et joyeuse habitude de peindre ses maisons. Depuis le brun rouge jusqu'au vert clair, en passant par les nuances du lilas, du rose et du gris, toutes les couleurs s'étalent gaiement sur les vieilles murailles qu'elles rajeunissent. (M. Du Camp, En Hollande : lettres à un ami, 1859)

L'existence d'une très forte corrélation entre en passant par figé et la structure syntaxique qui l'accueille est confirmée, par ailleurs, par des données statistiques que nous avons obtenues à partir d'une étude sur corpus ${ }^{6}$. En effet, si les emplois libres de en passant par n'apparaissent dans la structure tripartite que dans $12 \%$ des cas, la fréquence des emplois figés dans la même structure atteint $95 \%$. Cette observation met au jour une relative perte de liberté syntaxique de la séquence en passant par en emploi figé dans la mesure où un tel fonctionnement est fortement associé à une structure phrastique particulière. La possibilité d'avoir dans la même structure tripartite des emplois libres (concrets ou figurés), a dû jouer un rôle décisif dans l'évolution de l'expression en passant par, comme le suggèrent les exemples qui suivent :

(27) On voyait sur la paroi de cette chambre deux sillons parallèles et verticaux qui allaient d'un mur à l'autre en passant par la voûte où ils étaient profondément empreints, et qui donnaient l'idée de deux ornières. (V. Hugo, Quatre vingt-treize, 1874)

(28) Le territoire français est partagé en sept grands réseaux, dont cinq ont leur tête de ligne à Paris et deux - Alsace-Lorraine et midi - ne touchent pas la capitale. Il est plus facile, à cause de ce dispositif, de se rendre de l'est à l'ouest, ou du nord au sud en passant par Paris, qu'en prenant les voies transversales. (A. Albitreccia, Ce qu'il faut connaître des grands moyens de transport, 1931)

(29) Ainsi est faite l'histoire jusqu'à ce jour dominante; elle va de Bossuet à Karamsin en passant par l'abbé Pluche. Cette histoire a pour principe l'obéissance. (V. Hugo, William Shakespeare, 1864)

(30) Et en effet, plus de 30 millions de Français et Françaises pratiqueraient ce type de loisirs créatifs. Qui sont-ils ? L'éventail est large. Cela va des étudiants au budget serré qui cherchent la bonne affaire, à la jeune mère de famille qui confectionne les vêtements de son bébé, en passant par les jeunes cadres trentenaires qui courent les ateliers de la chaîne Natures et Découvertes pour apprendre à fabriquer leur crème hydratante bio. (Le Parisien, octobre 2010)

On est en présence ici d'une structure à la fois gérondive et tripartite. L'apparition dans la principale des formes fléchies des verbes aller et se rendre au sens premier et/ou figuré et la coréférence des sujets propre au gérondif nous empêchent de parler du figement. 
Rappelons que, selon nos données, en passant par figé apparaitt très rarement en dehors de la structure tripartite (dans $5 \%$ des cas). Il nous semble cependant que, même s'ils sont statistiquement marginaux, ces emplois ne doivent aucunement être négligés parce qu'ils sont révélateurs d'un stade de grammaticalisation plus avancé de en passant par. Il s'agit en effet de l'usage de en passant par figé dans des "séries énumératives", dont voici quelques exemples :

(31) L'engouement des auditeurs pour les grandes "voix ", sur l'antenne de France Culture et France Inter en passant par France Musique ou Radio Classique, ne faiblit pas. (Le Figaro, août 2010)

(32) Pour préparer les obsèques d'un de ses amis scénariste de cinéma, Barlow entre en contact avec ses homologues, les pompes funèbres pour êtres humains: les Célestes Pourpris. Cette entreprise aurait pu être imaginée par un Orwell à l'heure de Disneyland: la préparation des corps, le lieu de l'enterrement, en passant par le rituel et la musique, tout est à la carte, tout est pris en charge. Mourez, nous ferons le reste, disait un célèbre slogan. (Le Figaro, août 2010)

(33) «Ils ont des ouvertures sur différents aspects des métiers de service et de l'hôtellerie, serveur, maître d'hôtel, chef de rang, en passant par le bar. " (l'oral, c.p. A. Le Draoulec)

Ici, le statut fonctionnel de l'expression est encore plus prononcé : en passant par s'affranchit de la structure tripartite, les points extrêmes indispensables pour celle-ci n'étant plus explicités dans l'énoncé. Grâce à cette plus grande autonomie au niveau syntaxique, en passant par permet d'introduire un élément de l'énumération et peut se glisser dans des séries qui remplissent des fonctions syntaxiques différentes : sujet (32), COD (50), complément indirect (COI, complément de lieu, ou autre) (34), complément du nom (31), etc. :

(34) Ne parlons pas non plus des mille et une controverses entre les Ordres, des mesquineries provinciales de la hiérarchie internationale, des oscillations incessantes de gauche à droite et de droite à gauche, intégrismes, populismes et culturalismes divers, selon qu'on se retrouve à New York, au Japon, en passant par l'Afrique dévastée, le Brésil, Beyrouth, Mexico, Montréal ou la difficile Hollande. (Ph. Sollers, Le Secret, 1993)

Contrairement aux occurrences de en passant par figé dans la structure tripartite qui, en règle générale, peuvent être glosées par 'via', celles qui relèvent de l'emploi énumératif (31-34), se glosent plutôt à l'aide de 'y compris', 'ou bien', 'et'. L'interprétation de l'exemple (35) est beaucoup moins tranchée: sans la connaissance de la disposition géographique exacte de la Meije, des Écrins, du Pelvoux et du glacier des Violettes, on peut se demander si Gabriel est passé par ce dernier en faisant le Pelvoux ou si en passant par ne fait que rajouter un glacier dans la liste des glaciers parcourus :

(35) Gabriel lui, partait avec quelque imprudence sans dire sa destination et revenait illuminé d'avoir fait la Meije, les Écrins, le Pelvoux en passant par le glacier des Violettes. (J. Ollagnier, Main, 2008)

C'est le fait de savoir que le glacier des Violettes se situe au Pelvoux qui imposera la lecture spatiale. Il va de soi qu'il n'y aurait pas d'ambiguïté possible si à la place du SN le glacier des violettes on avait le glacier d'Orny, qui renvoie à un glacier se situant en Suisse.

Notons enfin que les emplois figés de en passant par, en particulier ceux qui figurent dans une construction tripartite, sont très proches de configurations de type : D'abord $X$, (et) puis/ensuite $Y$, et (enfin) $Z$ - dans le domaine temporel. Plusieurs travaux en analyse de discours ont montré que ces expressions peuvent perdre leur valeur temporelle pour intervenir au niveau de l'organisation discursive en tant que marqueurs d'intégration 
linéaire (voir notamment Dalmas 1998, Bras 2007, Bras \& Schnedecker 2009, Turco \& Coltier 1998, Lagae ce numéro). À l'instar de ces adverbiaux temporels qui, à la suite d'un processus de désémantisation, finissent par marquer l'ordre discursif, la séquence en passant par permet d'effectuer une sorte d'intégration linéaire au sein de la phrase, les emplois énumératifs représentant l'aboutissement de cette tendance. Cette possibilité n'existe évidemment pas pour les emplois libres.

3) Une troisième propriété vient à l'appui de la nécessité de distinguer les emplois libres et figés. En effet, en emploi libre, le gérondif du verbe passer (par) est régi par un verbe à la forme fléchie et, bien que la règle de coréférence ne soit pas absolue en français (cf. Halmøy 2003 : ch. VII), le sujet du gérondif est le plus souvent identique au sujet de la forme verbale régissante, comme dans l'exemple qui suit :

(36) Nous entrons dans le parc en passant par une majestueuse grille latérale. (Ph. Forest, L'enfant éternel, 1997)

Cette règle ne s'observe pas dans les emplois figés, la coréférence du sujet étant $a$ priori exclue autant dans la structure tripartite que dans la structure énumérative :

(37) Les divisions qui s'emparent de l'UMP traduisent aussi l'inquiétude croissante qui habite la majorité. De l'affaire Woerth à la difficile réforme des retraites, en passant par la perspective du remaniement gouvernemental annoncé pour novembre, la rentrée 2010 est la plus difficile pour la majorité depuis 2007. (Internet)

On voit dans cet exemple premièrement qu'il n'y a pas de verbe recteur pour en passant par et deuxièmement, qu'on ne peut plus identifier le sujet de passer : il est évident que ce n'est pas la rentrée 2010 qui passe par une perspective du remaniement gouvernemental. L'expression en passant par permet d'ajouter un élément dans la série des motifs de discorde au sein de la majorité parlementaire. La perte du sujet non seulement syntaxique, mais aussi sémantique est un indice de figement assez fiable. Il n'y a pas non plus de coréférence dans les séries énumératives (31-33). Ces observations vont toutes dans le sens de l'analyse proposée par Halmøy (2003 : 77-78) qui considère en passant par comme un cas de «figement prépositionnel» résultant du processus de grammaticalisation. D'après l'auteure, les gérondifs figés « ne se laissent pas rattacher à un constituant de la phrase » (idem, 77) et la règle de coréférence ne s'applique pas (idem, 115). Ce trait de fonctionnement est le plus marquant dans les titres constitués uniquement de la structure tripartite sans aucun verbe à la forme finie (38). Dans de tels contextes, tout éventuel rattachement de la structure en passant par à un verbe recteur est exclu :

(38) Du portier au digicode, en passant par le concierge. (titre sur www.paperblog.fr)

Les arguments avancés dans ce qui précède nous semblent suffisants pour fonder l'opposition entre les emplois libres et figés de la séquence en passant par. Il s'ensuit que cette expression présente en français contemporain un double comportement dans la mesure où dans certains contextes elle est syntaxiquement libre et sémantiquement transparente alors que dans d'autres elle apparaît comme syntaxiquement fort contrainte et sémantiquement opaque. Ajoutons cependant, à la suite de Gross (1996: 8), que " contrainte syntaxique et opacité sémantique sont de nature scalaire » et que parfois, il est difficile de poser des limites nettes entre les deux types d'emplois. Voici quelques exemples de ce genre :

(39) Tout en racontant à Étienne la mort de son ami Potice, il voyait défiler une succession d'images qui commençait à la mentonnière de sa grand-mère et finissait 
à sa propre pendaison en passant par l'absurde incident du caniche incongru. (R. Queneau, Le Chiendent, 1933)

(40) Les autorités historiques se trouveront aussi dans ces notes. On y verra enfin d'assez longs morceaux de mon itinéraire de Paris à Jérusalem, en passant par la Grèce. (F. de Chateaubriand, Les Martyrs ou le Triomphe de la religion chrétienne, 1810)

En y regardant de plus près, on constate qu'il y a des arguments pour les deux types de fonctionnement (libre et figé). Ainsi, en (40), il est évident que le trajet du locuteur passe nécessairement par la Grèce, mais ce n'est pas le déplacement proprement dit qui est décrit ici. En passant par la Grèce est à gloser plutôt par 'sans oublier la Grèce'. De même, en (39), la présence de commencer et finir suggère la lecture non figée, mais elle ne l'est pas vraiment.

Nous passerons dans la section suivante à la justification de notre deuxième hypothèse, selon laquelle en passant par fonctionne, dans ses emplois figés, comme une préposition complexe.

\subsection{En passant par - une nouvelle préposition complexe?}

L'objectif de cette section est d'examiner l'hypothèse selon laquelle en passant par a subi en diachronie un processus de grammaticalisation qui a transformé le gérondif du verbe passer suivi de par en une nouvelle préposition complexe. Rappelons que, pour valider cette hypothèse, il nous faut prouver l'absence des emplois qualifiés de figés dans des stades antérieurs de la langue et l'appartenance de en passant par à la classe des prépositions en français d'aujourd'hui.

Cette partie de l'étude s'appuiera sur deux corpus: l'un constitué à partir de la base textuelle Frantext (période 1500-2008), l'autre constitué à l'aide de GlossaNet à partir d'une sélection de journaux français allant de juillet 2010 à février 2011. La taille de ces deux corpus, le nombre d'occurrences de la séquence en passant par réparties par siècle ainsi que la taille de l'échantillon analysé ${ }^{7}$ figurent dans le tableau 1 ci-dessous:

Tableau 1. Caractérisation du corpus étudié

\begin{tabular}{|c|c|c|c|c|c|c|}
\hline Corpus & & $\begin{array}{l}\text { Nombre de } \\
\text { textes }\end{array}$ & $\begin{array}{l}\text { Nombre de } \\
\text { mots }\end{array}$ & $\begin{array}{l}\text { Nombre } \\
\text { d'occurrences de }\end{array}$ & $\begin{array}{l}\text { Fréquence } \\
\text { relative }^{8}\end{array}$ & Echantillon \\
\hline & XVI & (153 textes) & 5817432 & 20 & 3,43 & 20 \\
\hline & XVII & (572 textes) & 21840540 & 46 & 2,10 & 46 \\
\hline Frantext & XVIII & (554 textes) & 35396617 & 70 & 1,98 & 70 \\
\hline & XIX & (979 textes) & 71306804 & 228 & 3,20 & 228 \\
\hline & $\mathrm{XX}$ & $\begin{array}{l}(1790 \\
\text { textes) }\end{array}$ & 112426176 & 648 & 5,76 & 237 \\
\hline & GlossaNet & & & 789 & & 103 \\
\hline & Total & & & 1801 & & 704 \\
\hline
\end{tabular}


Les données de Frantext ont été recueillies dans le but d'examiner, période par période depuis le $16^{\mathrm{e}}$ siècle, la répartition des différents types d'emplois dégagés précédemment, d'observer leur éventuelle évolution et d'identifier le cas échéant le moment où en passant par figé commence à gagner en fréquence, voire à fonctionner comme une unité linguistique à part entière. Le deuxième corpus a été constitué pour évaluer le statut de en passant par de nos jours. Précisons enfin que, pour illustrer différents points de notre analyse, nous avons fait appel à quelques exemples construits et à Internet.

\subsubsection{Du gérondif à la préposition en passant par la collocation}

Nous commencerons par observer, sous forme de graphique, la répartition des emplois de en passant par entre les quatre structures identifiées, pour la période $16^{\mathrm{e}}-21^{\mathrm{e}}$ siècle : gérondive (libre - 41), tripartite (figée - 42), énumérative (figée - 43) et gérondive/ tripartite (semi-figée - 44) :

(41) Le cortège s'est rendu au Trocadéro en passant par Bercy.

(42) L'utilisation intensive d'Internet permet les pires débordements, de la délation à la diffamation en passant par l'injure sans risques. (Internet)

(43) Il a peint toute la cuisine en rouge, le sol, le plafond, les murs en passant par le chauffe-eau et le frigo.

(44) Les variations de couleurs, qui vont du beige au noir en passant par des tons de vert, de bleu et de rouille, s'obtiennent par la qualité de l'argile. (Internet)

\section{Graphique 1. Évolution des quatre types d'emplois de en passant par}

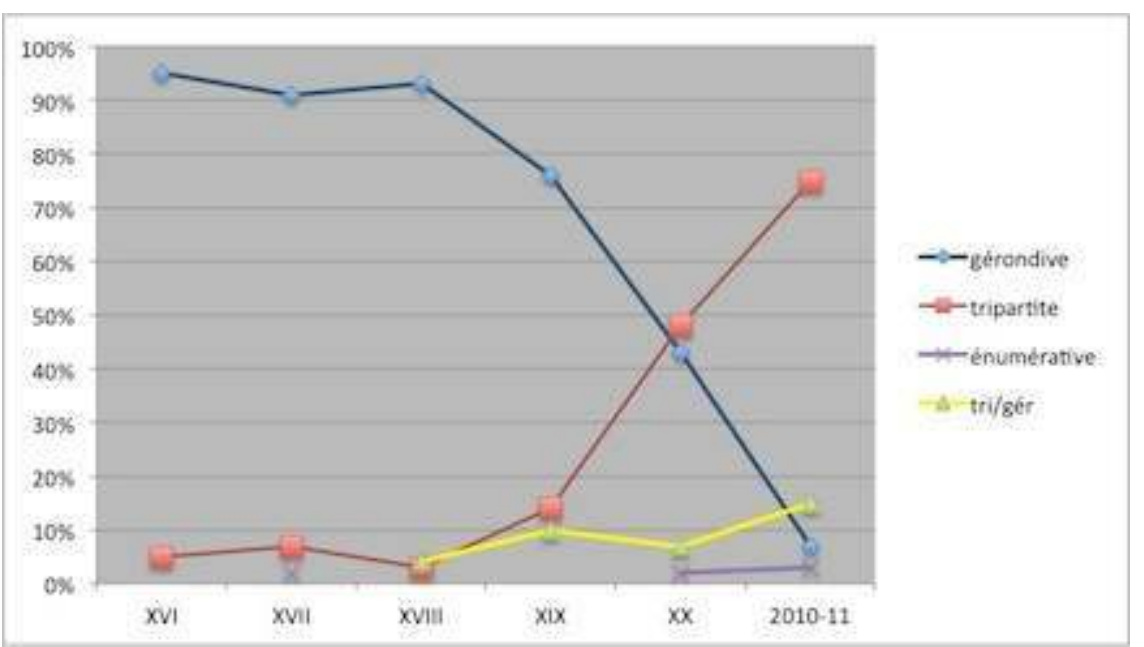

Ce graphique montre tout d'abord que la période entre 1500 et 1800 est marquée par une stabilité : en passant par connaît uniquement des emplois libres, concrets (45) et figurés (46) :

(45) Comment Gargantua courut après Gallimassue qui s'en estoit fuy, et comment en passant par la Champaigne il trouva encores les boys que sa grant jument avoit abbatuz, lesquelz il porta à Paris. (Anonyme, Les chroniques admirables, 1534)

(46) Aussi faut-il observer, qu'en apparence quelquefois l'action du theatre cesse, bien qu'en effet il ne soit pas ainsi. Ce qui arrive lors que le poëte prépare un incident qui doit éclatter dans la suitte, et dont il fait dire quelque chose avec adresse et comme en passant par l'un des acteurs. (Abbé d'Aubignac, La Pratique du théâtre, 1657) 
41 miers indices de l'évolution apparaissent au $18^{\mathrm{e}}$ siècle avec le relevé de quelques structures qui, sans être vraiment figées, ne sont pas entièrement transparentes du point de vue de leur interprétation sémantique :

(47) Je remarquerai ici que de Petersbourg à Pékin on trouverait à peine une montagne dans la route que les caravanes pourraient prendre par la Tartarie indépendante; et de Petersbourg aux extrémités de la France septentrionale, en passant par Dantzick, Hambourg, Amsterdam, on ne voit pas seulement une colline un peu haute. (Voltaire, Histoire de l'Empire de Russie sous Pierre le Grand, 1759)

L'évolution se confirme au $19^{\mathrm{e}}$ siècle : la courbe correspondant à la structure gérondive commence à descendre, alors que celle correspondant à la structure tripartite commence à monter. C'est à ce moment-là que la fréquence relative de en passant par augmente (voir l'avant-dernière colonne du Tableau 1 ci-dessus) ${ }^{9}$.

(48) Depuis la peau blanche et les cheveux roux de l'Anglais jusqu'au cuir bronzé et à la laine noire de l'Africain, en passant par les nuances intermédiaires café, cuivre et jaune d'or, toutes les variétés de l'espèce humaine se trouvent rassemblées là. ( $T$. Gautier, Voyage en Espagne, 1843)

(49) Là, sur une table entourée à distance d'un divan large et moelleux, tous les tabacs connus depuis le tabac jaune de Pétersbourg jusqu'au tabac noir du Sinaï, en passant par le maryland, le porto-ricco et le latakié, resplendissaient dans les pots de faïence craquelée qu'adorent les hollandais. (A. Dumas père, Le comte de MonteChristo, 1846)

Cette tendance s'affirme dans la période qui suit; en effet, c'est depuis les années mille neuf cent vingt que les emplois qualifiés ici de figés gagnent significativement en importance. Au $19^{\mathrm{e}}$ siècle la structure gérondive passe de $93 \%$ à $76 \%$ puis à $43 \%$ au $20^{\mathrm{e}}$. Inversement, la structure tripartie passe de $14 \%$ au $19^{\mathrm{e}}$ à $48 \%$ au $20^{\mathrm{e}}$ siècle. Enfin, dans le corpus journalistique $\mathrm{du} 21^{\mathrm{e}}$ siècle, la structure gérondive se réduit à $7 \%$, alors que la structure tripartite atteint $75 \%$ des emplois ${ }^{10}$. Les emplois figés de type énumératif (cf. $\S$ 2.1., ex. 31-33) n'apparaissent, selon nos données, qu'au $20^{\mathrm{ème}}$ siècle :

(50) Mon patchwork intérieur, que je me gardais bien d'exposer, juxtaposait des calotypes de barricades, en juin 1848 à Paris, et des clichés de bonzes en feu dans les rues de Saigon en 1961, en passant par les icônes de Blanqui, Lénine, Rosa Luxemburg, Bolivar, Victor Serge, Zapata, Beloyannis, Jean Moulin et Hô Chi Minh. (R. Debray, Loués soient nos seigneurs : une éducation politique, 1996)

Une synthèse des statistiques présentées dans le graphique 1 est donnée dans le tableau 2 :

Tableau 2. Répartition des emplois de en passant par en diachronie

\begin{tabular}{|l|l|l|l|l|}
\hline \multirow{2}{*}{ Période } & \multicolumn{4}{|l|}{ STRUCTURE } \\
\cline { 2 - 5 } & gérondive & tripartite & énumérative & tri/gér \\
\hline XVI & $95 \%$ & $5 \%$ & & \\
\hline XVII & $91 \%$ & $9 \%$ & & \\
\hline XVIII & $93 \%$ & $3 \%$ & & $4 \%$ \\
\hline XIX & $76 \%$ & $14 \%$ & & $10 \%$ \\
\hline
\end{tabular}




\begin{tabular}{|l|l|l|l|l|}
\hline XX & $43 \%$ & $48 \%$ & $2 \%$ & $7 \%$ \\
\hline GlossaNet & $7 \%$ & $75 \%$ & $3 \%$ & $15 \%$ \\
\hline
\end{tabular}
présente les propriétés citées par de nombreux spécialistes pour caractériser la grammaticalisation (cf. entre autres, Hopper 1991, Marchello-Nizia 1999, De Mulder 2001 et Fagard 2006b: 93 pour une synthèse): stratification, divergence, spécialisation, persistance, décatégorisation. On constate en effet que :

- tout au long de l'évolution de en passant par, la forme lexicale d'origine a continué à coexister en tant qu'élément autonome avec la forme grammaticalisée (divergence) ;

- le sens de la séquence devient de plus en plus opaque et général, d'où le développement d'un fonctionnement syntaxique particulier (spécialisation) ;

- les emplois figés gardent des traces du sémantisme respectif de passer et de par, ainsi que celles de la nature prédicative de passer (persistance) ; 
- en emploi figé, en passant par n'est plus soumis aux contraintes syntaxiques qui pèsent en général sur le gérondif (ex. pas de coréférence de sujet) tout en gardant intacte sa forme morphologique (décatégorisation). énumérative) où il apparaît, en passant par qualifié de figé est un élément invariable qui fonctionne comme un relateur (Pottier 1962), c'est-à-dire comme une expression qui met en relation un élément $\mathrm{A}$ et un élément $\mathrm{B}$, et qui sert à insérer son complément dans une construction de rang supérieur. Ce sont justement ces traits qui sont souvent utilisés pour définir la préposition (cf. Riegel et al, 1994, Fagard 2006a, b, De Mulder \& Stosic 2009). Précisons que dans des séries énumératives, tout en restant un « relateur ", en passant par semble plutôt coordonner des éléments du syntagme complexe où il apparait, comme le fait par exemple avec en (51) :

(51) J'ai rencontré Nicolas avec ses deux enfants. ( = Nicolas et ses deux enfants). cette différence importante que en passant par, du fait de son héritage prédicatif, a une plus grande capacité à s'insérer dans la structure de la phrase. De son côté, via a développé des emplois instrumentaux (52-53) qui, à l'heure actuelle, semblent assez marginaux dans le cas de en passant par (54):

(52) Pour écouter la radio via Internet, cliquez et écoutez Nederland.FM. (Internet)

(53) Bon à savoir : vous accédez à ces services via une connexion sécurisée. Toutes vos données restent confidentielles. (Internet)

(54) Il serait mieux de pouvoir ce (sic) connecter depuis le logiciel et non en passant par le site mobile. (Internet)

Il n'en reste pas moins que, d'un point de vue strictement distributionnel, les SPrép introduits par en passant par et via peuvent être coordonnés (55-56), ce qui prouve l'identité de fonctionnement syntaxique des deux éléments :

(55) A regarder de très très près comment le Japon peut envisager - voire si ce n'est déjà fait - de "détourner » le trafic de véhicules sortant du Japon en évitant des tests de radioactivité, en passant par l'Afrique, et notamment via le Sénégal. Un article suit dans le week-end, photos à l'appui... prises par moi-même. (Internet)

(56) Pour l'instant le mode de commande est un peu chaotique, en attendant que le livre entre dans les circuits de distribution classiques. COMMENT COMMANDER ? A l'heure actuelle, en passant par moi et via mail. (Internet)

A part les SPrép en via, rares sont les SPrép qui sont susceptibles d'être coordonnés avec ceux introduits par en passant par, à cause des particularités de son sémantisme mais aussi 
parce que la construction n'est pas au bout du parcours de grammaticalisation. Voici quelques exemples supplémentaires avec une autre préposition simple (depuis) et une préposition complexe figée (à partir de) :

(57) Visitez le site Alinea.fr afin de jeter un coup d'œil sur les boîtes de rangement et surtout les cubes de rangement modulables qu'il propose. (...) Ce sont des boîtes de rangement déco qui, en les intégrant dans votre intérieur, donneront un style design. Vous allez donc pouvoir ranger votre maison, depuis le salon et en passant par les chambres et la cuisine aussi. (Internet)

(58) Ainsi, à partir du sanscrit (langue commune indienne, identique au latin des Occidentaux) et en passant par l'étape des "prakrits » (les « moyens Indiens » où sont déjà sensibles les différences dialectales bases des futures langues), on est arrivé aux environs de l'an 1000 à la constitution des entités ethnolinguistiques actuelles. La stabilisation ethnolinguistique s'est donc faite en Inde à peu près à la même époque qu'en Europe. (Internet)

Au niveau sémantique, l'accession de l'expression en passant par à la catégorie de la préposition se traduit par une certaine désémantisation de ses constituants, ce dont témoigne l'affaiblissement des contraintes lexicales sur le choix des compléments. En même temps, le sens de l'expression en question n'est plus composite, les traits liés au mouvement s'estompent pour laisser la place à des traits plus abstraits permettant l'expression d'une relation fonctionnelle/syntaxique (réinterprétation sémantique).

Lorsqu'on tente de déterminer le degré de figement de l'expression en passant par, on se heurte à plusieurs difficultés. Tout d'abord, la grande majorité des tests utilisés à cet effet dans la littérature est conçue pour les prépositions complexes formées selon le modèle le plus productif : [Prép (Dét) Nom Prép] (cf. Pottier 1974, Leeman 2007, 2008, Adler 2001). Cela signifie que, dans le cas d'un figement, la manipulation du déterminant est impossible, la modification par un adjectif ne peut pas se faire, le remplacement du nom par un pronom, non plus. Ensuite, Adler (2001) a bien montré que certains de ces tests ne sont pas pertinents parce que même des expressions dont l'appartenance à la classe des prépositions est largement admise peuvent y répondre positivement. C'est par exemple le cas du test de l'insertion, qui consiste à insérer un élément à l'intérieur d'une préposition complexe. En effet, si les prépositions complexes comme à travers ou au pied de n'admettent aucune insertion, il y en a d'autres (ex. en dehors de, sous peine de, par rapport à , etc.) qui le font sans que cela remette en cause leur statut prépositionnel :

(59) Aussi acquiert-il très tôt renom et estime en dehors même de sa province natale. (Internet)

(60) À partir du 1er octobre 2008, le gilet de sécurité et le triangle de présignalisation seront obligatoires sous peine bien sûr de sanction. (Internet)

(61) Un autre chercheur, Rich Kay, a observé que le diamètre des deux conduits hypoglosses était, chez l'homme de Néandertal, aussi grand que le nôtre, par rapport évidemment aux dimensions de la cavité buccale. (Internet)

Appliqué à en passant par, le test de l'insertion donne un résultat positif :

(62) Livres Libres est une série d'émissions consacrée au livre sous toutes ses formes de son impression à son édition en passant évidemment par l'écriture. (Internet)

(63) Le léopard mange, dévore tout ce qui est en chair, de l'oiseau au guépard, en passant même par les grenouilles et les insectes les jours de disette! (Internet)

(64) Je côtoie aussi bien les professionnels que les étudiants en passant bien-sûr (sic ) par les enseignants. (Internet)

(65) La cinquième partie [de l'exposition] intitulée "la mesure du temps" sera consacrée aux instruments de la mesure du temps depuis les gnomons et les sabliers jusqu'aux horloges mécaniques en passant nécessairement par les clepsydres et les horloges hydrauliques. (Internet) 
$\mathrm{Au} \mathrm{vu}$ des exemples (59-65), on peut dire que le test de l'insertion ne remet pas nécessairement en question la possibilité de considérer en passant par comme une seule unité en français moderne. Les adverbes susceptibles d'être insérés entre en passant et par dans les emplois figés ne sont aucunement là pour modifier la réalisation de l'action de passer; il s'agit pour la plupart d'adverbes « exo-phrastiques » qui " représentent des traces de l'intervention du locuteur, qui commente tout ou partie de son énoncé ou de l'acte qui le produit» (Guimier 1996: 7). Cette restriction aux seuls adverbes exophrastiques et l'exclusion des adverbes de manière ne font que confirmer la perte des propriétés sémantiques et syntaxiques de passer. L'apparition d'un adverbe de manière entre en passant et par est en revanche un indice sûr de l'emploi libre de la structure en question, parce que les adverbes de ce type participent nécessairement à la construction du sens référentiel du prédicat verbal:

(66) Il allait s'accroupir devant le feu de charbon de bois, avalait son jus et remontait prendre son quart à la barre en passant prudemment par l'autre bord pour nous éviter. (B. Cendrars, Bourlinguer, 1948)

Rappelons également que toutes les prépositions complexes ne présentent pas le même degré de grammaticalisation, ce qui permet de distinguer les prépositions lexicales et les prépositions fonctionnelles. D’après Fagard $(2006 \mathrm{~b}: 92)$ : « les prépositions fonctionnelles ont un rôle plus syntaxique, une forme plus courte et un sens plus 'grammatical' ou général que les prépositions lexicales ». La délimitation entre les deux n'est pas toujours évidente, si bien qu'on parle plutôt d'un continuum de grammaticalité. Il en est de même du figement: certaines prépositions complexes sont plus figées que d'autres, d'où la difficulté, à maintes reprises soulignée dans la littérature, de circonscrire avec précision les contours de la classe des prépositions (cf. entre autres, Borillo 1997, Adler 2001, Melis 2003, Fagard 2006a, b, Fagard \& De Mulder 2007, Gross 2006, Leeman 2007, 2008). A défaut de critères fiables applicables à en passant par, nous ferons appel ici à l'augmentation de la fréquence, qui est considérée par certains spécialistes de la grammaticalisation comme fondamentale lorsqu'il s'agit d'identifier l'émergence d'une unité nouvelle, mais aussi d'évaluer son degré de figement (cf. entre autres, Bybee 1985, 2003, Bybee \& Hopper (eds) 2001, Fagard \& De Mulder 2007, Hoffmann 2005). L'idée sous-jacente à cette hypothèse est que plus un élément, un groupe d'éléments ou une structure sont récurrents dans l'usage, plus grandes sont les chances qu'ils soient stockés tels quels dans la mémoire des locuteurs et donc qu'ils deviennent des unités de langue à part entière. Dans ce sens, la grammaticalisation de certains éléments ou structures est très étroitement liée à leur usage, et plus précisément, à leur fréquence d'utilisation ${ }^{17}$. La fréquence d'usage peut donc être considérée comme un facteur crucial de changements sémantiques et fonctionnels. L'augmentation de la fréquence d'usage observée à propos de en passant par (cf. tableau 1 ci-dessus) indique très clairement qu'il s'agit, sinon d'une expression complètement figée, au moins d'une structure émergente en voie de grammaticalisation. Ce critère s'avère particulièrement pertinent lorsqu'il s'agit des emplois caractérisés ici de figés, dont la fréquence d'emploi est beaucoup plus importante que celle des emplois libres.

Compte tenu de son fonctionnement aujourd'hui, décrit dans ce qui précède, en passant par peut être considéré comme une préposition lexicale complexe dans les structures tripartites et énumératives où la coréférence du sujet est exclue. Elle garde cependant son fonctionnement d'expression libre (structure gérondive) et semi-figée (structure qualifiée 
de gérondive/figée), mais encore une fois, d'autres éléments ayant subi la grammaticalisation ont gardé, en parallèle, des emplois libres et/ou semi-figés.

\section{Conclusion}

Le en passant par en synchronie du français moderne et en diachronie $\left(16^{\mathrm{e}}-21^{\mathrm{e}}\right.$ siècle) nous a permis de mettre au jour sa double nature $:$ i) construction gérondive, libre et de sens transparent, en lien avec un prédicat recteur et ii) préposition complexe ayant atteint un degré de figement très important, et en tant que telle remplissant une fonction très particulière sur le plan syntaxique et présentant un comportement syntaxique différent de celui dans la construction gérondive. La valeur de la préposition complexe en passant par ne résulte plus du sens de ses constituants, le contenu lexical de base s'est considérablement effacé pour laisser la place à la prise en charge d'un contenu sémantique plus abstrait et d'une relation syntaxique spécifique au niveau de la phrase. Tous ces indices confirment que le changement catégoriel observé relève bien de la grammaticalisation. questions. S'il est par exemple évident que en passant par présente, pour des raisons sémantiques, une prédisposition à développer des emplois figés, prépositionnels, on peut se demander pourquoi cette structure s'est grammaticalisée, tandis que d'autres structures similaires n'ont pas connu une telle évolution (ex. en passant à travers, en rentrant de, en sautant sur, etc.). Pour répondre à cette question, il faudrait étudier plusieurs constructions parallèles - non grammaticalisées, mais aussi celles qui connaissent un degré de figement certain : (en) allant de, (en) partant de, etc. Il s'agit certes d'éléments du trajet cognitivement les plus saillants (point de départ, lieu de passage, jalon de parcours), mais est-ce une explication satisfaisante? Rappelons que le gérondif de passer connait également des emplois discursifs (En passant, j'attire votre attention sur la nécessité d'étudier le rapport entre 'en passant' et 'en passant par'). Comment expliquer la formation d'une préposition supplémentaire en français alors qu'il existe déjà par et via qui pourraient très bien assumer les emplois de en passant par figé ? Pourquoi de et par sont-ils « renforcés » par un verbe (aller, partir, passer) dans certains emplois et quelles sont les particularités des structures phrastiques où l'on rencontre de tels emplois?

Il serait tout aussi intéressant d'expliquer pourquoi, contrairement à un grand nombre de marqueurs spatiaux qui se sont grammaticalisés en prépositions ayant entre autre la valeur spatiale (au pied de, à côté de, chez, etc.), en passant par renie complètement son domaine d'origine pour n'avoir, une fois grammaticalisé, que des emplois abstraits/ fonctionnels dans les structures tripartite et énumérative. Le changement catégoriel s'avère donc lourd de conséquences sur les plans syntaxique et sémantique! On peut noter également que, encore une fois, c'est une construction à sémantisme spatial qui s'est grammaticalisée, plutôt qu'un marqueur provenant d'un domaine sémantique plus abstrait (mais lequel ?).

Cette étude remet également au premier plan la question du rapport entre le figement et la fréquence, utilisés souvent comme critères d'identification des structures grammaticalisées : faut-il définir le degré de grammaticalisation par la fréquence ou par le figement? Cette question rejoint celle de savoir quels critères il faut retenir pour définir les prépositions complexes, en synchronie mais aussi en diachronie.

Corela, HS-12 | 2012 


\section{BIBLIOGRAPHIE}

Adler, S., 2001. « Les locutions prépositives : questions de méthodologie et de définition », Travaux de linguistique 42-43, 157-170.

Aurnague, M., 2000. «Entrer par la petite porte, passer par des chemins de traverse : à propos de la préposition par et de la notion de « trajet » », Carnets de grammaire 7, 1-65.

Aurnague, M. \& Stosic, D., 2002. « La préposition par et l'expression du déplacement : vers une caractérisation sémantique et cognitive de la notion de « trajet » », Cahiers de lexicologie 81, 1-27.

Borillo, A., 1997. « Aide à l'identification des prépositions de temps et de lieu », Faits de langues 9, 173-184.

Borillo, A., 1998. L'expression de l'espace en français. Paris : Ophrys.

Bras, M., 2007. «French adverb d'abord and Discourse Structure », in M. Aurnague, J.M. Larrazabal \& K. Korta (eds), Language, Representation and Reasoning. Memorial Volume to Isabel Gomez Txurruka. Bilbao : Presses Universitaires du Pays Basque, 77-102.

Bras, M. \& Schnedecker, C., 2009. « Dans un (premier+second+nième) temps et les relations de discours : de l'élaboration à la contre-argumentation ». Colloque international Linguistic and Psycholinguistic Approaches to Text Structuring, Paris (France) 21-23 septembre 2009.

De Mulder, W., 2001. « La linguistique diachronique, les études sur la grammaticalisation et la sémantique du prototype : présentation ", Langue française 130, 9-32.

De Mulder, W. \& Stosic, D., 2009. « Présentation », Langages 173 (Approches récentes des prépositions ), 3-14.

Dalmas, M., 1998. « D'abord, et après ? Le marqueur d'intégration français et ses cousins germains ", in C. Schnedecker (ed), Les corrélats anaphoriques. Paris : Klincksieck, 75-95.

Detges, U. \& Waltereit, R., 2002. « Grammaticalization vs. Reanalysis. A semantic-pragmatic account of functional change in grammar », Zeitschrift für Sprachwissenschaft 21, 151-195.

Fagard, B., 2006a. Évolution sémantique des prépositions dans les langues romanes : phénomènes de grammaticalisation. Thèse de doctorat. Université Paris VII \& Université Rome III.

Fagard, B., 2006b. « La grammaticalisation en question : du latin aux langues romanes modernes », Modèles linguistiques 53, 91-110.

Fagard, B. \& De Mulder, W., 2007. « La formation des prépositions complexes : grammaticalisation ou lexicalisation? ", Langue française 156, 9-29.

Gross, G., 1996. Les expressions figées en français. Noms composés et autres locutions. Paris : Ophrys.

Gross, G., 2006. « Sur le statut des locutions prépositives », Modèles linguistiques 53, 33-50.

Guimier, C., 1996. Les adverbes du français : le cas des adverbes en -ment. Paris / Gap : Ophrys.

Halmøy, O., 2003. Le gérondif en français. Paris : Ophrys.

Haspelmath, M., 1998. « Does grammaticalization need reanalysis? », Studies in language 22-2, 49-85. 
Heine, B., Claudi, U. \& Hünnemeyer, F., 1991. Grammaticalization : A Conceptual Framework. Chicago : University of Chicago Press.

Hoffmann, S., 2005. Grammaticalization and English Complex Prepositions. Londres : Routledge.

Hopper, P., 1991. « On some Principles of Grammaticization », in E. Traugott \& B. Heine (eds), Approaches to Grammaticalization. Amsterdam/Philadelphia : John Benjamins, 17-35.

Hopper, P. \& Traugott, E., 1993. Grammaticalization. Cambridge : Cambridge University Press.

Lagae, V., (ce numéro), « Marqueurs du point de départ spatial et temporel antéposés : une comparaison de depuis, dès et à partir de ", Corela.

Langacker, R.W., 1977. « Syntactic reanalysis », in C.N. Li (ed), Mechanisms of Syntactic Change. Austin / London : University of Texas Press, 57-139.

Laur, D., 1991. Sémantique du déplacement et de la localisation en français : une étude des verbes, des prépositions et de leurs relations dans la phrase simple. Thèse de doctorat. Toulouse : Université de Toulouse-Le Mirail.

Leeman, D., 2007. « De la préposition à la locution prépositionnelle », Modèles linguistiques 55, 7-15.

Leeman, D., 2008. « Les locutions en sous : description du français et traductions ", L'information grammaticale $117,9-12$.

Lehmann, Ch., 1995. Thoughts on Grammaticalization. München : Lincom Europa.

Marchello-Nizia, Ch., 1999. Le français en diachronie : douze siècles d'évolution. Paris : Ophrys.

Meillet, A., [1912] 1948. Linguistique historique et linguistique générale. Paris : Champion.

Melis, L., 2003. La préposition en français. Paris : Ophrys.

Pottier, B., 1962. Systématique des éléments de relation. Paris : Klincksieck.

Pottier, B., 1974. Linguistique générale, théorie et description. Paris : Klincksieck.

Prévost, S., 2006. « Grammaticalisation, lexicalisation et dégrammaticalisation : des relations complexes », Cahiers de praxématique 46, 121-139.

Prévost, S. \& Fagard, B., 2007. « Grammaticalisation et lexicalisation : la formation d'expressions complexes ", Langue française 156, 3-8.

Prévost, S. \& Fagard, B. (eds), 2007. Langue française 156. Grammaticalisation et lexicalisation : la formation d'expressions complexes.

Riegel, M., Pellat, J.-C. \& Rioul, R., 1994. Grammaire méthodique du français. Paris : PUF.

Stosic, D., 2002. « Par » et « à travers » dans l'expression des relations spatiales : comparaison entre le français et le serbo-croate. Thèse de doctorat. Toulouse : Université de Toulouse-Le Mirail.

Svorou, S., 1994. The Grammar of Space. Amsterdam / Philaldelphie : John Benjamins.

Turco, G., Coltier, D., 1988. « Des agents doubles de l'organisation textuelle : les marqueurs d'intégration linéaire », Pratiques 57, 40-60.

\section{Dictionnaires}

DHLF (Dictionnaire historique de la langue française). Paris : Dictionnaires Le Robert, 1992.

Dictionnaire Larousse français-anglais, http://www.larousse.fr/dictionnaires/francais-anglais 
Le Nouveau Petit Robert, J. Rey-Debove \& A. Rey. Paris : Dictionnaires Le Robert, 1994.

Le dictionnaire des expressions et locutions, A. Ray \& S. Chantreau. Paris : Dictionnaires Le Robert, 1993.

Le Robert électronique (CD-ROM)

Lexis, Dictionnaire de la langue française, J. Dubois. Paris : Larousse, 1994.

Sylang, http://dict.sylang.com/en-passant-par+anglais

TLFi (Trésor de la langue française informatisé), http://atilf.atilf.fr/tlf.htm

\title{
Corpus utilisés
}

\author{
Frantext (http://www.frantext.fr/) \\ GlossaNet (http://glossa.fltr.ucl.ac.be/) \\ Internet (via Google)
}

\section{NOTES}

1. Mes plus sincères remerciements aux deux relecteurs anonymes et à B. Fagard pour leurs commentaires et remarques sur une version antérieure de cet article. Leurs conseils judicieux m'ont permis d'améliorer considérablement mon travail.

2. Les exemples littéraires sont extraits de la base Frantext, ceux de la presse sont relevés via l'outil GlossaNet (voir les références à la fin de l'article). L'absence d'indication de la source signifie qu'il s'agit d'un exemple construit.

3. Les critères opposant les emplois «libres" aux emplois «figés"sont exposés dans le paragraphe 2.1. ci-dessous.

4. Du fait de son absence dans la plupart des dictionnaires mono- ou bilingues, en passant par pose souvent problème aux traducteurs, ce qui en fait un objet de discussions récurrent dans des forums (voir par exemple forum.wordreference.com).

5. Cela n'explique cependant pas POURQUOI c'est en passant par qui se grammaticalise et non en rentrant de, en passant à travers ou en montant sur, pour ne prendre que quelques exemples de marqueurs spatiaux sémantiquement tout aussi compatibles que passer et par. Pour comprendre ce qui favorise la grammaticalisation de certaines structures spatiales, il faudrait étudier plusieurs constructions parallèles. Nous remercions B. Fagard d'avoir attiré notre attention sur cet aspect de la problématique.

6. Voir la section 2.2. pour la présentation de notre corpus de travail.

7. Nous avons analysé tous les exemples relevés pour les $16^{\mathrm{e}}, 17^{\mathrm{e}}, 18^{\mathrm{e}}$ et $19^{\mathrm{e}}$ siècles, et une sélection aléatoire d'exemples pour les $20^{\mathrm{e}}$ et $21^{\mathrm{e}}$ siècles.

8. En nombre d'occurrences pour 1000000 de mots.

9. Le fait que la ligne qui correspond au $16^{\mathrm{e}}$ siècle a une fréquence plus élevée que celle correspondant au $19^{\mathrm{e}}$ siècle est à mettre en rapport avec l'insuffisance du corpus pour le $16^{\mathrm{e}}(20$ occurrences au total, dont 7 viennent d'un même auteur - B. Palissy).

10. Sans exclure la possibilité que l'écart mis en évidence puisse être dû en partie au genre journalistique, nous estimons qu'il confirme une tendance générale assez nette en français moderne concernant le fonctionnement de l'expression en passant par.

11. Que certaines structures complexes ayant un usage très fréquent dans le discours finissent par devenir des unités de langue est un phénomène linguistique assez courant et déjà étudié par 
de nombreux chercheurs. Pour un aperçu de ces travaux, voir par exemple Bybee \& Hopper (2001:8-9).

12. Pour un aperçu des chaînes de grammaticalisation aboutissant à des prépositions lexicales, dans une perspective typologique, voir Fagard (2006b : 95).

13. Aurnague (2000) avance un certain nombre d'arguments en faveur de la lexicalisation de la structure passer par.

14. Faute de place, nous ne ferons pas de développement particulier sur l'opposition entre la lexicalisation et la grammaticalisation, les deux processus étant souvent intimement liés lors de l'évolution d'un élément linguistique. Pour à la fois une bonne synthèse et une réflexion approfondie sur la question, voir Prévost (2006), Prévost \& Fagard (éds) (2007).

15. La « réanalyse » accompagne souvent la grammaticalisation et constitue un processus très important dans l'évolution des langues. Faute de place, nous ne développerons pas ce point ici (pour plus de détails, voir entre autres Langacker 1977, Lehmann 1995, Haspelmath 1998, Detges \& Waltereit 2002).

16. Nous ne nous prononçons pas ici sur la question de savoir si le degré de grammaticalisation est à définir par la fréquence ou par le figement. Le poids des deux critères mériterait une étude à part.

17. Hoffmann (2005) montre, cependant, sur l'exemple des prépositions complexes en anglais, que la grammaticalisation et la fréquence d'usage ne vont pas toujours de pair.

\section{RÉSUMÉS}

L'objectif de cet article est d'examiner le fonctionnement morpho-syntaxique et sémantique de l'expression en passant par en français en synchronie et en diachronie (1500-2011). Une étude sur corpus fait ressortir d'importantes différences de fonctionnement entre les emplois du début de la période prise en compte et ceux du français moderne. Ces différences suggèrent une évolution assez nette qui aboutit à la coexistence, en français contemporain, d'emplois «libres » correspondant au gérondif du verbe passer suivi d'un syntagme prépositionnel en par (ex. J'ai vu une boulangerie en passant par cette rue) et de la forme figée en passant par, proche par son comportement des prépositions, statut qui ne lui est reconnu ni par les lexicographes ni par les linguistes (ex. Il a tout lu de Montaigne à Gary en passant par Gautier et Balzac). Pour expliquer ce double fonctionnement en français moderne, nous étudions l'hypothèse d'une grammaticalisation en cours, et la testons au moyen d'une étude sur corpus synchronique et diachronique. De manière plus générale, cette analyse met au jour les conséquences sémantiques et fonctionnelles du changement catégoriel que la forme gérondive du verbe de déplacement passer est en train de subir.

In this paper, we analyse the morpho-syntactic and semantic functioning of the expression en passant par in French, in synchrony as well as in diachrony (1500-2011). A corpus-based study brings clearly out two very different behaviours of this expression respectively in the sixteenth century and in Modern French. All differences revealed by our analysis suggest an obvious evolution that results in a coexistence in contemporary French of a free construction corresponding to the gerund of the verb passer 'to pass' followed by a PP in par (e.g. J'ai vu une boulangerie en passant par cette rue) with the non compositional expression en passant par, similar in many ways to prepositions (e.g. Il a tout lu de Montaigne à Gary en passant par Gautier et Balzac). 
Neither lexicographers nor linguists acknowledge this prepositional status of en passant par. In order to explain this double behaviour of en passant par in Modern French, we study the hypothesis of an ongoing grammaticalization, and test it on the basis of data drawn from written literary and journalistic texts. More generally, this study brings to the fore the semantic and functional consequences of the categorial change that currently affects the gerund of the motion verb passer in French when followed by par.

INDEX

Keywords : preposition, grammaticalization, semantics, space

Mots-clés : préposition, grammaticalisation, espace, sémantique, figement

\section{AUTEUR}

\section{DEJAN STOSIC}

Grammatica (EA 4521), Université d'Artois 